Open Access

\title{
Clinical outcomes of immunoglobulin use in solid organ transplant recipients: protocol for a systematic review and meta-analysis
}

Juthaporn Cowan ${ }^{1 *}$, Brian Hutton², Nicholas Fergusson², Alexandria Bennett ${ }^{2}$, Jason Tay ${ }^{2,3}$, D. William Cameron ${ }^{1,2}$ and Greg A. Knoll ${ }^{2,4}$

\begin{abstract}
Background: Transplantation improves survival and the quality of life of patients with end-stage organ failure. Infection, due to surgical issues, host factors such as diabetes, immunosuppression, and hypogammaglobulinemia, is a major post-transplant complication. Clinical outcomes of prophylaxis or treatment of hypogammaglobulinemia in solid organ transplant recipients are not well established and are in need of further study.

Methods/design: We will conduct a systematic review of studies investigating clinically relevant outcomes of immunoglobulin use either as prophylaxis or treatment of hypogammaglobulinemia after solid organ transplantation. Both randomized and non-randomized studies (excluding case reports and case series of less than 20 subjects) will be included. Outcomes of interest will include the overall rate of infection, hospital admission, hospital length of stay, intensive care unit admission, 1-year all-cause mortality, incidence of acute organ rejection, allograft survival within 1 year, and adverse events. We will search MEDLINE, Embase, the Cochrane Central Register of Controlled Trials, Transplant library, and the International Clinical Trials Registry Platform for randomized and non-randomized studies on adult solid organ transplant patients who received prophylactic immunoglobulin or immunoglobulin treatment. Two reviewers will conduct all screening and data collection independently. We will assess study level of risk of bias using the Cochrane Risk of Bias Assessment Tool for randomized controlled trials and for non-randomized studies. If meta-analysis of outcome data is deemed appropriate, we will use random effects models to combine data for continuous and dichotomous measures.
\end{abstract}

Discussion: The results of this systematic review may inform guideline development for measuring immunoglobulin level and use of immunoglobulin in solid organ transplant patients and highlight areas for further research.

Systematic review registration: PROSPERO CRD42015017620

Keywords: Immunoglobulin, Hypogammaglobulinemia, Solid organ transplantation, Systematic review

\footnotetext{
* Correspondence: jcowan@toh.on.ca

'Division of Infectious Diseases, Department of Medicine, University of

Ottawa, Ottawa, Canada

Full list of author information is available at the end of the article
}

\section{Biomed Central}

(c) 2015 Cowan et al. Open Access This article is distributed under the terms of the Creative Commons Attribution 4.0 International License (http://creativecommons.org/licenses/by/4.0/), which permits unrestricted use, distribution, and reproduction in any medium, provided you give appropriate credit to the original author(s) and the source, provide a link to the Creative Commons license, and indicate if changes were made. The Creative Commons Public Domain Dedication waiver (http://creativecommons.org/publicdomain/zero/1.0/) applies to the data made available in this article, unless otherwise stated. 


\section{Background}

Hypogammaglobulinemia (gamma-immunoglobulin (IgG) $<700 \mathrm{mg} / \mathrm{dL}$ or $7 \mathrm{~g} / \mathrm{L}$ ) is a complication of solid organ transplantation (SOT) that occurs after immunosuppressive therapy or treatment of organ rejection [1]. The reported incidence of hypogammaglobulinemia among kidney transplant recipients is between 40 and $60 \%$ [2-5]. This is similar to the overall rate of hypogammaglobulinemia in all SOT recipients [4]. The rate, duration, and severity of hypogammaglobulinemia are further modified by a variety of additional factors including host and donor characteristics, presence of graft rejection, and its accompanying use of immunosuppressive therapy [6-8].

Immunosuppression is vital in transplantation; however, it also increases risk of infection. Hypogammaglobulinemia is associated with an increased rate of bacterial infection as compared to transplant recipients without hypogammaglobulinemia (15 vs $5 \%$ ) [9]. Posttransplant hypogammaglobulinemia is an independent risk factor for overall (hazard ratio (HR) 2.03; 95 \% confidence interval (CI) 1.39-2.96) and bacterial infection (HR 2.68; 95 \% CI 1.66-4.34) in kidney transplant recipients [3]. Hypogammaglobulinemia is also an independent risk factor for Clostridium difficile-associated colitis in heart transplantation recipients [10]. Severe hypogammaglobulinemia ( $\operatorname{IgG}<4 \mathrm{~g} / \mathrm{L}$ ) is also a risk factor for death following SOT [4]. It is not known whether the increased risk of mortality is solely related to severe infection as a result of hypogammaglobulinemia in this population.

Intravenous immunoglobulin (IVIG) is prepared from pooled plasma involving thousands of healthy blood donors. The large donor pool ensures a diversity of antibody specificities to a wide spectrum of antigens and microbial pathogens [11]. The use of IVIG or any form of immunoglobulin in hypogammaglobulinemia after solid organ transplantation has not been well explored. A retrospective study looking at IVIG therapy and survival post-transplantation has been recently published [12]. This study showed that increasing immunoglobulin levels with IVIG did not have any impact on survival. However, the study was limited by its retrospective design and small sample size.

IVIG has been used in highly sensitized kidney transplant recipients to permit successful transplantation and reduce the risk of antibody-mediated rejection [13]. IVIG is thought to be effective in this setting by blocking complement activation, neutralizing donor-specific antibodies via anti-idiotypic HLA antibodies, and downregulating HLA antibody production by donor-specific B-cells.

To date, clinical outcomes related to the prophylactic or therapeutic use of immunoglobulin in SOT recipients have not been analyzed. The planned systematic review will address this knowledge gap in order to establish the current state of the evidence on this topic.

\section{Research objectives}

We will conduct a systematic review to evaluate clinical outcomes associated with the use of immunoglobulin after solid organ transplantation.

\section{Methods}

\section{Search strategy}

A comprehensive electronic search will be conducted in MEDLINE (1950 to September 2015), Embase (1950 to September 2015), and the Cochrane Central Register of Controlled Trials (September 2015). The assistance of a medical librarian experienced in systematic reviews will be used in designing the search strategy. A structured search strategy will be based on controlled vocabulary and relevant key terms and will be broad to prioritize sensitivity (Additional file 1). Language of publication will not be restricted, and the draft search will be peer reviewed by a second medical librarian according to PRESS criteria [14]. The references of included articles and existing reviews will be scanned for additional studies that were not identified by the search. Transplant library search (www.transplantevidence.com) will be performed in order to identify studies that were published as conference proceedings or only in abstract forms. We will search the International Clinical Trials Registry Platform (www.who. int/ictrp/en/) to identify incomplete or unreported studies.

\section{Study screening and inclusion}

Using the results of our comprehensive search strategy, we will obtain title and abstract from all references. First titles and abstracts (stage 1) and then full-texts of potentially relevant studies (stage 2) will be screened by two reviewers independently following short pilot exercises to identify and address any inconsistencies in the application of screening criteria. The inclusion and exclusion criteria used for each stage of screening are outlined below. If no abstract is available for a given citation, the full-text will be obtained unless the article can be confidently excluded by its title alone. In general, if there is any doubt as to whether a study should be excluded, the study will proceed to the full-text screen to reduce the likelihood of excluding a relevant study. A third party will reconcile any disagreements if discussion between the two reviewers cannot determine the article's inclusion status. The process of study selection will be summarized using a PRISMA flow diagram [15].

\section{Study eligibility criteria}

Studies will be selected according to the criteria outlined in Table 1. 
Table 1 PICOS breakdown of study eligibility criteria

\begin{tabular}{ll}
\hline Category & Description of criteria \\
\hline Population & Adults undergoing solid organ transplant (including the \\
& heart, lung, liver, kidney, intestine, and pancreas) \\
Intervention & Polyvalent immunoglobulin prophylaxis or polyvalent \\
& immunoglobulin treatment without restriction to \\
& dosage, frequency, timing, and route of administration \\
Comparator(s) & Placebo or no treatment. Patients may receive \\
& antimicrobial prophylaxis, which should be the same in \\
& both intervention and control arms \\
Outcome(s) & Primary outcome: \\
1) Overall rate of infection & 2) Rate of infection based on severity: non-severe \\
infection, severe infection, and serious/fatal infection \\
Secondary outcomes: \\
1) Hospital admission, initial length of stay, and number \\
of re-admissions in the first year after transplantation; \\
2) ICU admission; \\
3) 1-year all-cause mortality; \\
4) Incidence of acute rejection; \\
5) Allograft survival at 1 year after transplantation \\
6) Adverse events graded by severity [21] \\
Both randomized controlled trials and non-randomized \\
studies including case series with a minimum of 20 \\
cases will be included. Case reports and studies that \\
only focus on immunoglobulin use for de-sensitization \\
purposes will be excluded
\end{tabular}

\section{Data extraction}

Each eligible study will undergo a standardized data extraction process by two reviewers independently using a pre-designed form designed in Microsoft Excel (Microsoft Corporation, Seattle, WA, USA). Any discrepancies will be documented, discussed, and adjudicated by a third party. Prior to extraction of all study data, piloting of the form will first be conducted upon a small number of studies.

Information pertaining to study identification (author, year of publication, number and location of centers, funding, and journal); study design (type of study, sample size, eligibility criteria, and length of follow-up); aggregate patient characteristics (age, gender, duration of end organ damage prior to transplantation, type of transplantation, immunosuppressive regimen, comorbidities, onset of hypogammaglobulinemia, duration of hypogammaglobulinemia, treatment with immunoglobulin therapy either intravenously or subcutaneously, dose of immunoglobulin, and duration of treatment); and infection outcomes (the overall rate of infection as well as the rates of non-severe, severe, and serious/fatal infections) will be extracted. Hypogammaglobulinemia is defined as IgG level of less than $7 \mathrm{~g} / \mathrm{L}$. Severe hypogammaglobulinemia is defined as IgG level <4 g/L [4]. A serious/fatal infection will be considered to be any infection that required admission to intensive care unit or result in death. A severe infection will be considered any infection that required intravenous antimicrobial therapy and/or hospitalization. A nonsevere infection will be considered any infection that does not meet criteria for severe or serious/fatal infection [16, 17]. If available, etiologic agent of infection will also be recorded as gram-positive, gram-negative, or anaerobic bacteria; Candida spp.; filamentous fungi; or viruses. Site of infection will be recorded as central nervous system, respiratory tract, gastrointestinal, genitourinary, skin, soft tissue, or blood stream infection related/ non-related to catheter. Infection at other sites not specified above will be recorded as others. We will use individual study's definitions of infectious outcomes if they do not align with the set definition above.

\section{Risk of bias assessment}

Risk of bias in the randomized trials will be evaluated using the Cochrane Risk of Bias Assessment Tool [18]. Studies will be assessed based on the domains of randomization, generation of allocation sequence, allocation concealment, blinding, follow-up, and reporting. Non-randomized studies will be evaluated using the ACROBAT-NRSI [19] based on study design, confounding, reporting, and directness of evidence. Results from risk of bias assessments will be presented in their entirety. They will also be summarized narratively and may also inform sensitivity analyses. If there are sufficient numbers of trials (e.g., $\geq$ ten studies), we will construct a funnel plot to assess for possible publication bias.

\section{Data analysis}

For studies assessing the effect of immunoglobulin treatment, we will report the overall rates of infection, nonsevere infection, severe infection, and serious/fatal infection per 100 patient-days in patients receiving immunoglobulin treatment for secondary hypogammaglobulinemia. We will also report stratified infection rates for patients with severe and non-severe hypogammaglobulinemia. Type of infection (e.g., bacterial, viral) and site of infection will also be reported. For studies assessing the effect of prophylactic immunoglobulin, we will report overall rates of infection and mortality, as well as adverse events.

We will compare overall rates of infection in the intervention and control (no IVIG) groups of each study and report the corresponding relative risk measures along with the corresponding with $95 \%$ confidence interval. A relative risk of less than 1 will suggest a beneficial effect of intravenous immunoglobulin, while a relative risk of greater than 1 will suggest a harmful effect. Prior to conduct of meta-analyses, team members will review characteristics of the included studies and their patient populations such as type of study, outcome measures, and organ transplanted to establish the extent to which they appear homogeneous. The Cochrane Q/chi-square test and $I^{2}$ statistic will also be calculated to evaluate heterogeneity. We will use $I^{2}$ cutoff of $\geq 75 \%$ to be considered as considerable heterogeneity [20]. Where meta-analysis is 
considered to be appropriate, we will pool data using a random effects model. Meta-analysis of non-randomized studies will be performed separately from meta-analysis of randomized trials. A systematic narrative synthesis will be provided with information presented in the text and tables to summarize and explain the characteristics and findings of the included studies. The narrative synthesis will explore the relationship and findings both within and between the included studies, in line with the guidance from the Centre for Reviews and Dissemination [15].

We will perform several a priori sensitivity analyses to understand the data and to identify any subpopulations that may benefit from the use of intravenous immunoglobulin. These analyses will include stratification of studies according to baseline immunoglobulin level (IgG $\leq 4$, IgG $>4$ but $<7$, IgG $\geq 7 \mathrm{~g} / \mathrm{L}$ ), intent of immunoglobulin use (prophylaxis vs treatment), immunosuppressive regimen used (e.g., cyclosporin vs tacrolimus, induction vs no induction), and organ type (e.g., liver, kidney).

\section{Reporting of the review}

We will report our findings in accordance with guidance of the PRISMA statement [15] and its checklist. A completed copy of the checklist will be provided in a supplement to the main report (Additional file 2).

\section{Discussion}

In this systematic review, we will assess the treatment outcome of hypogammaglobulinemia post solid organ transplantation. We anticipate several challenges in this work. These will include the possibility of considerable study heterogeneity in terms of organ type, immunosuppressive regimen, study design, and outcomes reported. The availability of high-quality studies addressing this topic may also be limited. Studies may concentrate on a particular type of solid organ transplantation, in which case the data will be pooled and analyzed within the same type of transplantation. This may limit the generalizability to other types of solid organ transplantation but may provide for a better-focused interpretation.

Despite several anticipated challenges, our systematic review will provide important insights into the effects of immunoglobulin replacement therapy or prophylactic therapy in solid organ transplant recipients. The results may have a direct impact on current practice and practice guidelines. The data will also serve as a starting point for further research.

\section{Additional files}

Additional file 1: Search strategy. Serch terms and databases are outlined. (DOCX $15.2 \mathrm{~kb}$ )

Additional file 2: PRISMA-P checklist. Report of compliance with standard reporting guideline. (DOC $84 \mathrm{~kb}$ )

\section{Abbreviations}

HLA: human leukocyte antigen; IgG: gamma-immunoglobulin;

IVIG: intravenous immunoglobulin; SOT: solid organ transplantation.

\section{Competing interests}

The listed authors do have not any relevant competing interests.

\section{Authors' contributions}

GK is the guarantor. All authors conceived and designed the review. JC drafted the protocol. $\mathrm{BH}$ and GK critically reviewed and revised the protocol. $A B$ and NF will perform the study selection and data extraction. JC will reconcile any discrepancies in all steps of data collection. JC, BH, and GK will perform data interpretation and manuscript preparation. BH, JT, DWC, and GK will be consulted with the interpretation of results and preparation of the manuscript. All authors read and approved the final manuscript.

\section{Funding source}

This study is funded by the Department of Medicine Developmental Research Grant, University of Ottawa. The funder has no role in developing the review protocol.

\section{Author details}

${ }^{1}$ Division of Infectious Diseases, Department of Medicine, University of Ottawa, Ottawa, Canada. ${ }^{2}$ Clinical Epidemiology Program, Ottawa Hospital Research Institute, Ottawa, Canada. ${ }^{3}$ Blood and Marrow Transplant Program, The Ottawa Hospital, Ottawa, Canada. ${ }^{4}$ Renal Transplantation, Division of Nephrology, Department of Medicine, University of Ottawa, Ottawa, Canada.

Received: 1 September 2015 Accepted: 9 November 2015

Published online: 19 November 2015

\section{References}

1. Carbone J, del Pozo N, Gallego A, Sarmiento E. Immunological risk factors for infection after immunosuppressive and biologic therapies. Expert Rev Anti Infect Ther. 2011;9(4):405-13.

2. Broeders EN, Wissing KM, Hazzan M, Ghisdal L, Hoang AD, Noel C, et al. Evolution of immunoglobulin and mannose binding protein levels after renal transplantation: association with infectious complications. Transpl Int. 2008;21(1):57-64.

3. Fernandez-Ruiz M, Lopez-Medrano F, Varela-Pena P, Lora-Pablos D, Garcia-Reyne A, Gonzalez E, et al. Monitoring of immunoglobulin levels identifies kidney transplant recipients at high risk of infection. Am J Transplant. 2012;12(10):2763-73

4. Florescu DF, Kalil AC, Qiu F, Schmidt CM, Sandkovsky U. What is the impact of hypogammaglobulinemia on the rate of infections and survival in solid organ transplantation? A meta-analysis. Am J Transplant 2013;13(10):2601-10.

5. Mawhorter S, Yamani MH. Hypogammaglobulinemia and infection risk in solid organ transplant recipients. Curr Opin Organ Transplant. 2008:13(6):581-5.

6. Legris T, Picard C, Moal V, Burtey S, Loundou A, Purgus R, et al. Humoral immunity after kidney transplantation: impact of two randomized immunosuppressive protocols. Ann Transplant. 2013:18:622-34.

7. Rentenaar RJ, van Diepen FN, Meijer RT, Surachno S, Wilmink JM, Schellekens PT, et al. Immune responsiveness in renal transplant recipients: mycophenolic acid severely depresses humoral immunity in vivo. Kidney Int. 2002;62(1):319-28.

8. Meier-Kriesche HU, Friedman G, Jacobs M, Mulgaonkar S, Vaghela M, Kaplan B. Infectious complications in geriatric renal transplant patients: comparison of two immunosuppressive protocols. Transplantation. 1999;68(10):1496-502.

9. Norlin AC, Sairafi D, Mattsson J, Ljungman P, Ringden O, Remberger M. Allogeneic stem cell transplantation: low immunoglobulin levels associated with decreased survival. Bone Marrow Transplant. 2008;41(3):267-73.

10. Munoz P, Giannella M, Alcala L, Sarmiento E, Fernandez Yanez J, Palomo J, et al. Clostridium difficile-associated diarrhea in heart transplant recipients: is hypogammaglobulinemia the answer? J Heart Lung Transplant. 2007:26(9):907-14.

11. Kazatchkine MD, Kaveri SV. Immunomodulation of autoimmune and inflammatory diseases with intravenous immune globulin. N Engl J Med. 2001;345(10):747-55. 
12. Florescu DF, Kalil AC, Qiu F, Grant W, Morris MC, Schmidt CM, et al. Does increasing immunoglobulin levels impact survival in solid organ transplant recipients with hypogammaglobulinemia? Clin Transplant. 2014;28(11):1249-55.

13. Shehata N, Palda VA, Meyer RM, Blydt-Hansen TD, Campbell P, Cardella C, et al. The use of immunoglobulin therapy for patients undergoing solid organ transplantation: an evidence-based practice guideline. Transfus Med Rev. 2010;24 Suppl 1:S7-S27.

14. Sampson M, McGowan J, Cogo E, Grimshaw J, Moher D, Lefebvre C. An evidence-based practice guideline for the peer review of electronic search strategies. J Clin Epidemiol. 2009;62(9):944-52.

15. Moher D, Liberati A, Tetzlaff J, Altman DG, PRISMA Group. Preferred reporting items for systematic reviews and meta-analyses: the PRISMA statement. J Clin Epidemiol. 2009;62(10):1006-12.

16. Duraisingham SS, Buckland M, Dempster J, Lorenzo L, Grigoriadou S, Longhurst HJ. Primary vs. secondary antibody deficiency: clinical features and infection outcomes of immunoglobulin replacement. PLoS One. 2014;9(6):e100324.

17. Definitions of Inf Severity.doc - Definitions_of_Inf_Severity.pdf. Available at: https://web.emmes.com/study/bmt2/public/Definition/Definitions_of_Inf_ Severity.pdf. Accessed 9/28/2015.

18. Higgins JPT, Altman DG, Gøtzsche PC, Jüni P, Moher D, Oxman AD, et al. The Cochrane Collaboration's tool for assessing risk of bias in randomised trials. BMJ. 2011;343:d5928.

19. Sterne JAC, Higgins JPT, Reeves BC on behalf of the development group for ACROBAT-NRSI. A Cochrane Risk Of Bias Assessment Tool: for non-randomized studies of interventions (ACROBAT-NRSI), Version 1.0.0, 24 September 2014 Available from http://www.riskofbias.info. Accessed 9/28/2015.

20. 9.5.2 Identifying and measuring heterogeneity. Available at: http//handbook cochrane.org/chapter_9/9_5_2_identifying_and_measuring_heterogeneity.htm. Accessed 9/29/2015.

21. Common terminology criteria for adverse events (CTCAE) - CTCAE_4.03_201006-14_QuickReference_5x7.pdf. Available at: http://evs.nci.nih.gov/ftp1/CTCAE/ CTCAE_4.03_2010-06-14_QuickReference_5x7.pdf. Accessed 9/24/2015.

\section{Submit your next manuscript to BioMed Central and take full advantage of:}

- Convenient online submission

- Thorough peer review

- No space constraints or color figure charges

- Immediate publication on acceptance

- Inclusion in PubMed, CAS, Scopus and Google Scholar

- Research which is freely available for redistribution 